Para enlazar con este artículo / To link to this article:

http://dx.doi.org/10.14198/fem.2017.30.01

Para citar este artículo / To cite this article:

Berger, Éléonore. «Tuer la mère. Quand luce irigaray rencontre Angelica Liddell». En Eva García-Ferrón y Cristina Ros-Berenguer (coords.), Dramaturgia femenina actual. De 1986 a 2016. Feminismo/s, 30 (diciembre 2017): 21-30, DOI: 10.14198/fem.2017.30.01

\title{
TUER LA MÈRE QUAND LUCE IRIGARAY RENCONTRE ANGÉLICA LIDDELL
}

\author{
KILL THE MOTHER \\ WHEN LUCE IRIGARAY MEETS ANGÉLICA LIDDELL
}

\author{
Éléonore BERGER \\ Universidad de la Sorbonne Nouvelle-Paris 3 \\ eleo.berger@gmail.com \\ orcid.org/0000-0001-8128-5332
}

\section{Résumé}

Dans les années 70, des psychanalystes comme Julia Kristeva et Luce Irigaray repensent la maternité et la figure de la mère, à rebours de la pensée freudienne, afin de poser les bases d'une nouvelle appréhension et une nouvelle construction de l'identité féminine. Quarante ans de féminisme plus tard, Angélica Liddell hurle sa haine des femmes et de la figure maternelle sur les scènes européennes. Cet article a pour but de faire se rencontrer ces deux formes de pensées a priori radicalement opposées, notamment en comparant «Le corps-à-corps avec la mère» de Luce Irigaray et la lutte à mort que Liddell organise dans Tout le ciel au-dessus de la terre. Il s'agit de voir comment Liddell, peut-être contre son gré, travaille le matricide originel dénoncé par Irigaray pour délivrer une version violemment moderne de l'affranchissement du désir féminin.

Mots-clefs: Figure maternelle et figure féminine, Identité, Angélica Liddell, Luce Irirgaray, Féminisme.

\begin{abstract}
During the seventies, psychoanalysts such as Julia Kristeva and Luce Irigaray went against Freud's vision of both maternity and the figure of the mother by creating a new way of understanding these concepts to allow a different construction of feminine identity. Forty years of feminism later, the playwright and performer Angélica Liddell shouts her hatred of women and the figure of the mother on every European stage.
\end{abstract}

Feminismo/s 30, diciembre 2017, pp. 21-30

Los contenidos de la revista se publican bajo una licencia de Creative Commons Reconocimiento 4.0 Internacional (CC BY 4.0) 
This article aims to bring these two schools of thought, which at first glance appear diametrically opposed, together by comparing Irigaray's «Le corps-à-corps avec la mère» and the struggle shown by Liddell in Tout le ciel au-dessus de la terre. This paper intends to highlight how Liddell, possibly against her will, works with the original matricide illustrated by Irigaray and creates a violently new version of the female's freedom of desire.

Keywords: Mother's figure and feminine figure, Identity, Angélica Liddell, Luce Irigaray, Feminism. 


\section{INTRODUCTION}

«FOR ALL MOTHERS! FUCK YOU! MOTHER!» (Liddell 2013, 55). C'est sans équivoque qu'Angélica Liddell apostrophe la figure maternelle dans son spectacle présenté en 2013, Tout le ciel au-dessus de la terre (le syndrome de Wendy). Avec la radicalité et l'énergie empreinte de violence et d'éclatement qui caractérisent son œuvre depuis ses débuts, la dramaturge et sa compagnie Atra Bilis construisent une réflexion sur le passage de la vie adolescente à la vie adulte en mêlant le mythe de Peter Pan à la tuerie perpétrée à Utoya en juillet 2011. Le monologue qui occupe la dernière heure de ce spectacle permet le déploiement de la parole d'Angélica Liddell, qui quitte le personnage Wendy pour briser la barrière de la pudeur en son propre nom et déverser sur scène sa haine dirigée à l'encontre de «toutes les mères». En cela, son processus créateur vient conforter la constatation que fait Luce Irigaray dans «Le corps-à-corps avec la mère», une conférence qu'elle a donnée en 1980 à Montréal dans le cadre du cinquième colloque québécois sur la santé mentale organisé autour du thème des «femmes et la folie» et qui sert de point de départ de notre réflexion sur la portée féministe ambiguë de l'œuvre d'Angélica Liddell. Une partie de la réflexion d'Irigaray, dans cette conférence, se fait anthropologique: elle tente de reconstruire une forme de généalogie de la domination masculine, assurée par la main-mise faite sur le corps de la femme, le désir pour elle et son désir à elle. Elle part de cette hypothèse, s'inscrivant immédiatement à rebours de la pensée freudienne: «ce qui apparaît dans les faits les plus quotidiens comme dans l'ensemble de notre société et de notre culture, c'est que celles-ci fonctionnent originairement sur un matricide» (23). La haine que Liddell voue au monde entier n'épargne personne, mais se concentre particulièrement sur les figures féminines qu'elle rejette et dont elle regrette de faire partie dans son journal La Fiancée du fossoyeur: «[...] pardonne-moi de parler des femmes en étant femme [...] ça ne diminue en rien ma misogynie» $(2014,162)$. Cette misogynie revendiquée fait d'elle une personnalité souvent contestée, voire détestée. Dans You are my destiny (Le viol de Lucrèce) (2014), elle prend le point de vue de Tarquin, prenant de revers toute une tradition d'interprétation du mythe et excitant l'ire des spectateurs et plus particulièrement des spectatrices; 
Fabienne Pascaud écrit par exemple qu' «autour du viol de la sage et trop belle Lucrèce par Tarquin, dans la Rome antique du VIe siècle avant Jésus-Christ, elle [Angélica Liddell] a orchestré une toxique variation qui condamne et légitime à la fois le violeur».

Provocatrice et subversive, Liddell bouleverse les lignes d'interprétation. Le matricide est chez elle entièrement consommé, elle le consacre lorsqu'elle se désigne comme «née du sexe d'une détraquée mentale» dans la mesure où elle cherche par tous les moyens à s'éloigner de cette figure quand Irigaray prône une réunion avec celle-ci. Folie, féminisme, maternité et création sont des entités que Liddell fait jouer en permanence, notamment dans son exploration des limites et représentations du corps féminin. On peut donc supposer que le rapport conflictuel qu'entretient la dramaturge avec les figures féminines, et par extension avec les différents mouvements féministes dont elle se désolidarise, trouve un point d'ancrage dans le rapport conflictuel qu'elle entretient avec la figure maternelle.

\section{2. «MOI, SOEUR JUMELLE DE LILITH, ENNEMIE DES ACCOUCHEMENTS, DÉMON DANS LE DÉSERT [...]» (LIDDELL 2014, 163)}

Nous avons à veiller à autre chose: ne pas retuer la mère qui a été immolée à l'origine de notre culture. Il s'agit de lui redonner la vie, à cette mère, à notre mère en nous, et entre nous. Il faut que nous refusions que son désir soit anéanti par la loi du père. Nous devons lui donner droit au plaisir, à la jouissance, à la passion, lui redonner droit à la parole, parfois aux cris et à la colère. (Irigaray 30)

La figure paternelle est presque absente des œuvres d'Angélica Liddell, et l'on peut supposer que c'est la dramaturge elle-même qui endosse le rôle d'exécutrice de cette «loi du père» en consacrant le matricide. Déjà dans le «Triptyque de l'affliction», trois pièces sur le thème de la dégradation familiale (Monsieur et Madame Palavrakis (2001), Once upon a time in West Asphixia (2002) et Hysterica Passio (2003)), elle avait amorcé ce travail de démolition des figures parentales. Avec Lesiones incompatibles con la vida (2003): un spectacle de trente minutes où elle déclare ne pas vouloir d'enfants, elle passe pour la première fois de la fiction à la confession et prend en charge la parole à la première personne. Quelques années plus tard, dans Tout le ciel au-dessus de la terre, elle reprend une fois encore ce dispositif confessionnel en confondant les paroles du personnage de Wendy et le brut aveu de ses pensées qui jaillissent en logorrhée vindicative. Laccusation portée contre les mères fustige les «suppléments de dignité»: 
WENDY.- [...] Être mère, c'est le must. Être mère, ça rapporte tous les suppléments de dignité.

Dès que leur ventre se met à les démanger, elles réclament tous les suppléments de dignité.

Tu peux être la dernière des ordures, le simple fait d'être mère te rapporte tous les suppléments de dignité.

Tu peux être stupide et mauvaise, le simple fait d'être mère te rapporte tous les suppléments de dignité.

EN MAJUSCULES

Tu peux utiliser tes enfants pour te défendre, faire du chantage aux sentiments, le simple fait d'être mère te rapporte tous les suppléments de dignité. (68)

Ainsi la figure maternelle devient l'allégorie de l'hypocrisie du monde que la dramaturge ne cesse de dénoncer. En appuyant sur la fonction utilitariste à l'échelle sociale de l'image de mère, Liddell opère une déconstruction -par la désacralisation- du mythe de la femme-mère, cette figure de Marie ancrée dans la culture occidentale faisant de la maternité un accomplissement pour le sujet féminin. Irigaray pense qu'il est «nécessaire, pour ne pas être complice du meurtre de la mère, que nous affirmions qu'il existe une généalogie de femmes» (29-30). Pour Liddell, cette généalogie est un cycle dont il s'agit plutôt de s'affranchir: tuer la mère, c'est justement s'éloigner de la tare, de la «détraquée mentale». Elle lie ainsi la folie de la mère et la folie de la fille, rendant la première responsable de la seconde au coeur d'un mouvement cyclique aussi indéfectible que pessimiste:

Après avoir accouché, les femmes n'ont rien d'autre, les mères pétrissent leurs filles avec ce qu'il y a de pire en elles pour que l'histoire se répète. La fille de Wendy, la fille de la fille de Wendy, la fille de la fille de la fille de Wendy, la fille de la fille de la fille de la fille de Wendy... (70)

En effet, sa singularité se veut violemment coupée de toute origine et c'est ce qu'elle exprime dans son journal La Fiancée du fossoyeur:

Dans le ventre de ma mère, j'étais déjà brisée. Les yeux, les bras et les jambes mélangées comme des morceaux de boeuf dans un bouillon prophétique. [...] Quel genre d'homme peut trouver son origine dans un paysage, alors qu'il n'y a d'autre origine qu'un ventre débordant de liquide fécal. [...] Le moindre conflit me donne la nausée. Ce doit être une séquelle. Laffrontement me ramène à la division originelle de mes extrémités dans le sein maternel. Je ne le supporte pas. (91)

A l'origine ne se trouve pas une sécurité, un lieu protecteur, mais bien une division. Division qui par la suite engendre une monstruosité chronique et de transmission: «Les mères font de leurs filles leurs jumelles nauséabondes, sans même le faire exprès, sans même s'en rendre compte, elles pétrissent 
avec ce qu'il y a de pire en elles» déclare Liddell dans Tout le ciel au-dessus de la terre (68). Irigaray se prend à rêver à la fin de sa conférence à une «femme célébrant l'eucharistie avec sa mère [...] [qui] pourrait être délivrée de toute haine ou ingratitude vis-à-vis de sa généalogie maternelle» (33). Mais Liddell ne cherche pas cette libération car, au contraire, elle travaille avec l'aliénation. Il s'agit pour elle de rompre le cycle de transmission généalogique pour faire quelque chose d'autre de cette «tare»; de la folie congénitale, elle bascule alors vers une appropriation de diverses formes de folies qu'elle utilise à des fins créatrices. Elle ne travaille en effet qu'à partir de matériaux qui entretiennent une intimité essentielle avec des formes de folie: la douleur, le meurtre, l'idiotie, le mal de vivre, la bile noire dont sa compagnie porte le nom. En cela, elle semble incarner l'être de douleur dépeint par la psychanalyste Julia Kristeva dans son essai sur la littérature et l'abjection:

La douleur comme lieu du sujet. Là où il advient, où il se différencie du chaos. Limite incandescente, insupportable entre dedans et dehors, moi et autre. Saisie première, fugace: «douleur», «peur», mots ultimes visant cette crête où le sens bascule dans les sens, l'«intime» dans «les nerfs». Lêtre comme mal-être. (165)

Le mal-être pourtant n'est jamais chez Liddell un apitoiement, à la plainte se substitue une forme de dissection poétique et scientifique. De ses émotions-symptômes, elle déduit des syndromes. Tout le ciel au-dessus de la terre constitue une série des réflexions, constructions, variations autour du «syndrome de Wendy». Elle choisit de prendre le contre-pied en n'essayant jamais de se présenter sous l'apparat de la raison ou du raisonnable, travailler avec la folie lui permet de travailler avec une condition féminine pour laquelle elle ne prend pas les armes mais plutôt contre laquelle elle s'arme:

Tout comme j'ai conscience de la mortalité ou de la douleur, j'ai conscience - brutalement conscience - d'être femme. Je ne peux pas éviter de me sentir femme. C'est ancré en moi, je ne peux pas m'en défaire. Et cela implique des tas de choses à supporter, comme ces petits rituels d'humiliation qui nous sont imposés par le simple fait d'être femme. C'est pour moi insurmontable. Alors je dois transformer la douleur en quelque chose d'autre: quelque chose de beau. Non pas que je trouve de la beauté dans l'horreur, mais j'ai besoin de transformer l'horreur pour survivre. $(2012,45)$

Il s'agit alors pour elle non seulement de renverser ce qu'elle considère comme le privilège moral hypocrite de la figure maternelle, mais surtout de ne pas faire advenir cette fonction maternelle. 


\section{3. «JE PRÉFÈRE LES ENFANTS SANS ENFANTS. C'EST CE QUE JE PRÉFÈRE. LES ENFANTS SANS ENFANTS» (LIDDELL 2013, 67)}

C'est là toute la problématique du «syndrome de Wendy»: comment échapper à cette figure maternelle, symbole de l'abjection du monde des adultes? Comment soi-même, étant femme, ne pas devenir mère? Pour Luce Irigaray, la réponse est claire: la femme est indissociable de son rôle de mère, mais celle-ci ne doit pas être considérée uniquement par le prisme biologique. Être mère c'est aussi et surtout être créatrice, donner naissance à une œuvre au sens large: «nous procréons et créons autre chose que des enfants: de l'amour, du désir, du langage, de l'art [...]» (Irigaray 27). Liddell, toute mère de ses œuvres qu'elle est, affirme pourtant une singularité particulière, que ce soit métaphoriquement ou concrètement car elle se refuse à la fonction reproductrice:

PETER. - Vous jouez toutes à être mères. Vous adorez être mères.

WENDY. - Je ne veux être la mère de personne.

$[\ldots]$

PETER. - Les mères sont des raclures, des salopes, des vraies raclures!

WENDY. - Où as-tu mal, Peter?

PETER. - Laisse-moi, ce n'est pas ce genre de douleur! Je peux très bien faire sans mère. Je n'ai pas besoin d'une idiote de mère.

WENDY. - Je suis Wendy, je suis Wendy, je suis Wendy, je suis Wendy, je suis Wendy, je suis Wendy.... (19-20)

A cette fonction, elle trouve deux alternatives possibles. Dans le long monologue qu'elle performe en 2003, Lesiones incompatibles con la vida, Liddell déclare qu'elle ne veut pas d'enfants et utilise pour se représenter la métaphore d'une épidémie de ressentiment. Cette notion de «contagion» est intéressante en ce qu'elle constitue une alternative à celle de «reproduction»: la transmission s'effectue de côté, par endroits, comme un virus. D'un point de vue poétique, l'œuvre de Liddell peut être abordée sous ce prisme épidémique. Elle transmet le ressentiment et la haine car elle dépasse le cadre du théâtre en mettant en place un dispositif de création traversé par ses expériences personnelles et ses émotions face au monde. De plus en plus, journal intime, confession et pièce de théâtre se confondent et la personne contamine le personnage. Cette confusion des cadres génériques, tissu de contagion, permet la création d'un lien entre traumatisme personnel et histoire collective. Cela se produit entre pédophilie, peur de vieillir et drame d'Utoya dans le cas de Tout le ciel au-dessus de la terre: les jeunes garçons tués sur l'île deviennent l'objet du désir de Wendy. En creusant sa singularité et maniant l'intime, le je, Liddell trouve une première alternative au modèle reproductif. Mais l'on peut considérer que sa position de colère, de violence et de rejet des figures féminines est en elle-même 
une réinterprétation de ce schéma de construction de l'identité. Hélène Rouch, biologiste travaillant sur les limites de l'identité, se penche sur le rapport au placenta, ou plutôt, au placenta en lui-même. Pour elle, il est la membrane par excellence, permettant de penser la subjectivité, toujours en rapport à l'autre. Alors que chez Irigaray le placenta apparaît comme un gouffre terrifiant car écarté de la femme, il est chez Rouch un espace d'accueil. Elle remarque notamment qu'au niveau des cellules, il n'y a pas systématiquement des mécanismes de contre-agression: mais plutôt que la membrane peut accepter le corps étranger. Elle remet ainsi la notion d'identité qui repose sur l'exclusion de l'autre, et sur laquelle Irigaray s'appuie, peut-être inconsciemment. Le placenta comme «tiers»: c'est aussi une réponse aux psychanalystes, elle donne une autre idée de la mère, et de la supposée fusion naturelle entre la mère et l'enfant. Elle montre bien que et l'enfant et la mère sont dans la perte. Elle met ainsi en lumière l'archaïsme auquel s'accroche encore la pensée d'Irigaray tout en se débattant pour s'en extirper. Irigaray dénonce l'image de la mère que l'absence de corps-à-corps crée: «La mère est devenue monstre dévorant, par effet en retour de la consommation aveugle d'elle dans son ventre» (27) mais ne voit le positif que l'on peut trouver dans cet éloignement. Liddell développe elle aussi le rapport de la maternité avec la dévoration, mais en inversant ce rapport dans Tout le ciel au-dessus de la terre: "Je secrète à toute heure un lait sulfureux / qui ne nourrit pas» (57). Pour elle, la mère ne nourrit pas, elle ne se nourrit pas. Elle, symbole de la reproduction, devient une figure de la stérilité. Cette figure paradoxale permet alors le développement d'un personnage féminin en lutte avec sa condition, un personnage pour qui la maternité est tout à la fois un apport, un supplément, et pourtant un manque, une absence, un estomac vide qui demande à être comblé. En quelque sorte, Liddell, dans sa radicalité, obéit au programme d'Irigaray, elle «prend acte» de la coupure du cordon.

4. «JE SUIS VÊTUE DE CHAIR ET DE RIEN D'AUTRE» (LIDDELL 2014, 78)

Luce Irigaray lance un appel à la fin de sa conférence:

Nous avons aussi à trouver, retrouver, inventer, découvrir, les mots, les phrases, qui disent le rapport le plus archaïque et le plus actuel au corps de la mère, à notre corps, les phrases qui traduisent le lien entre son corps, le nôtre, celui de nos filles. Nous avons à découvrir un langage qui ne se substitue pas au corpsà-corps, ainsi que tente de le faire la langue paternelle mais qui l'accompagne, des paroles qui ne barrent pas le corporel mais qui parlent «corporel». (31)

Il s'agit de contrer ce matricide originel, de créer des liens entre toutes les femmes, d'encourager la sororité par un engagement concret du corps et du 
langage. Elle propose une alternative intéressante en préconisant et décrivant des conditions concrètes de production de ce nouveau langage: il faut alors parler en donnant le sein, parler dans l'échange amoureux, toujours parler pour vivre différemment un rapport au corps. Pour consacrer le matricide plutôt que le conjurer, Liddell n'en construit pas moins un lien corps/langage neuf. Ce lien est assuré d'une part par la pratique de la performance qui accompagne tous ses spectacles, et d'autre part par celle d'une diction particulière de ses textes qui confine à la profération car les paroles sont criées, le rythme est particulier et souvent accompagné d'une forte musique. Irigaray s'interrogeant sur le corps féminin réactive l'ancestrale comparaison de la mère à la terre. L'homme-territoire serait alors la frontière du langage, la délimitation qui oublie la fertilité de la terre. Cette métaphore permet de penser le problème que soulève Irigaray en termes géographiques et politiques de territorialisation. Des penseurs comme Bernard Debarbieux rappelle le rôle des «hauts lieux»: des lieux qui rappelle la présence de la terre et son pouvoir irrépressible d'échappée et de construction nouvelle, échappant au pouvoir de territorialisation des hommes. Ces «hauts lieux» sont des montagnes, des fractures dans l'espace physique qui rappellent la terre où nous posons nos pieds. De la même manière, le nombril est un lieu déhiscent, une cicatrice permettant le repli vers la mère-terre; et la scène est pour Liddell l'endroit de la vérité, son haut lieu faisant se rencontrer corps, espace et langage dans la vocifération et la performance. Pour Liddell, c'est la stérilité qui est un mode nouveau d'affirmation du corps en scène, corps désirant plus que désirable. Là où Irigaray pense substitution ou continuité «œuvre»/«enfant» en ne dissociant jamais création et procréation, Liddell pense une alternative radicale: «Soit tu as des enfants, soit tu te consacres à la pensée». Pour elle, la procréation est une impasse: «Durant neuf mois, les membres se sont mal combinés à cause du retard mental de ma mère, [...] et c'est finalement une créature aberrante, non viable, incapable de survivre à l'hostilité du monde qui a vu le jour» (2013, 91). Irigaray encourage à «découvrir la singularité de notre jouissance», Liddell explore et expose sa singularité dans la jouissance. Elle le fait par le biais de la performance à l'ouverture de Tout le ciel au-dessus de la terre, le spectacle s'ouvrant sur un rituel masturbatoire d'une durée d'environ quinze minutes. La performeuse se masturbe sur un monticule de terre, dans le silence le plus total, déjouant immédiatement barrières de la pudeur et de la décence pour mettre en lumière un corps féminin esseulé et en jouissance. En cela, elle répond bien paradoxalement à l'injonction d'Irigaray: «A travers tout ça, ce que nous avons à faire (mais il ne s'agit pas de faire l'un avant l'autre), c'est de découvrir notre identité sexuelle, c'est-à-dire la singularité de notre auto-érotisme, de notre 
narcissisme, la singularité de notre homosexualité» (32). Tout le ciel au-dessus de la terre organise une variation sur ce désir:

Les êtres que j'aime sont tellement petits,

tellement pâles,

tellement enfants

que mon corps répond comme celui d'une mère mais incongrue.

Je sécrète à toute heure un lait sulfureux

qui ne nourrit pas.

[...]

Moi, je me masturbe.

Face à la souffrance, je me masturbe.

Pas de sentiments. Pas d'amour. (91)

En mettant en avant masturbation et désir pédophile, voire nécrophile, Liddell occupe le terrain de la provocation, de la volonté de se constituer en corps désirant singulier. Elle emploie les codes féministes des années 70 en les dévoyant pour créer une œuvre hybride. Mère, mais «incongrue», Liddell développe spectacle après spectacle un rapport à la maternité marqué par le rejet, la haine, et la contradiction. Elle crée, tout en sortant du schéma traditionnel de procréation. Il s'agit pour elle de faire œuvre autrement, c'est-à-dire d'être femme autrement.

\section{RÉFÉRENCES BIBLIOGRAPHIQUES}

Debarbieux, Bernard. «Le lieu, le territoire et trois figures de rhétorique». Espace Géographique 24.2 (1995): 97-112.

Irigaray, Luce. «Le corps-à-corps avec la mère». Sexes et parentés. Paris: Les Editions de Minuit, 1987.

Kristeva, Julia. Pouvoirs de l'horreur. Essai sur l'abjection. Paris: Seuil, 1980.

Liddell, Angélica. La Maison de la force. Tétralogie du sang. Trad. Christilla Vasserot. Paris: Les Solitaires Intempestifs, 2012.

Liddell, Angélica. Tout le ciel au-dessus de la terre (el sindrome de Wendy). Trad. Christilla Vasserot. Paris: Les Solitaires Intempestifs, 2013.

Liddell, Angélica. You are my destiny (Le viol de Lucrèce). Le cycle des résurrections. Trad. Christilla Vasserot. Paris: Les Solitaires Intempestifs, 2014.

Liddell, Angélica. La Fiancée du fossoyeur. Le cycle des résurrections. Trad. Christilla Vasserot. Paris: Les Solitaires Intempestifs, 2014.

Pascaud, Fabienne. «Entre douleur et plaisir, les tortures scéniques d'Angelica Liddell». Télérama (2014).

Rouch, Hélène. «Le placenta comme tiers». Langages 21.85 (1987): 71-79. 